\title{
IL-10 is critical for Th2 responses in a murine model of allergic dermatitis
}

\author{
Dhafer Laouini, Harri Alenius, Paul Bryce, Hans Oettgen, Erdyni Tsitsikov, \\ and Raif S. Geha
}

Division of Immunology, Children's Hospital and the Department of Pediatrics, Harvard Medical School, Boston, Massachusetts, USA

\begin{abstract}
We found that mechanical injury to mouse skin, which can be caused by tape stripping, results in rapid induction of IL-10 mRNA. IL-10-/- mice were used to examine the role of IL-10 in a mouse model of allergic dermatitis induced by epicutaneous (EC) sensitization with OVA on tape-stripped skin. Skin infiltration by eosinophils and expression of eotaxin, IL-4, and IL-5 mRNA in OVA-sensitized skin sites were severely diminished in IL-10-/- mice. Following in vitro stimulation with OVA, splenocytes from EC-sensitized IL-10 ${ }^{-/-}$mice secreted significantly less IL-4, but significantly more IFN- $\gamma$, than splenocytes from WT controls. A similar skewing in cytokine secretion profile was observed in the splenocytes of IL-10 $1 /-$ mice immunized intraperitoneally with OVA. IL-10 $10^{-/-}$APCs skewed the in vitro response of OVA $T$ cell receptor (TCR) transgenic $T$ cells towards Th1. Examination of the Th response of WT and IL-10 ${ }^{-/-}$mice immunized with OVA-pulsed WT or IL-10 $10^{-/}$DCs revealed that both DCs and T cells participate in IL-10 skewing of the Th2 response in vivo. These results suggest that IL-10 plays an important role in the Th2 response to antigen and in the development of skin eosinophilia in a murine model of allergic dermatitis.
\end{abstract}

J. Clin. Invest. 112:1058-1066 (2003). doi:10.1172/JCI200318246.

\section{Introduction}

IL-10 is secreted by a wide variety of cell types, including Th cells, monocytes, macrophages, mast cells, eosinophils, and keratinocytes (1). IL-10 has been reported to be a global suppressor of immune responses as well as an immunoregulator of the Th cell response (2). In vitro studies have shown that IL-10 can inhibit the production of proinflammatory cytokines by APCs (IL-1, TNF- $\alpha$, IL-6, IL-12, GM-CSF) (3-6). IL-10 also inhibits $T$ cell proliferation to antigen and superantigen (7). IL-10 production occurs late after $\mathrm{T}$ cell activation in comparison with other cytokines. This is thought to represent an autocrine regulation of $\mathrm{T}$ cell responses (8). The immunoregulatory role of IL-10 appears to be complex. Several studies using protozoan infection models have shown that IL-10 plays an important role in balancing the protective and pathological immune responses during intracellular parasitic infection. Treatment of mice with anti-IL-10 leads to rapid control of Listeria within few days of infection (9). IL-10 $0^{-/-}$mice are resistant to infection by several organisms that include

Received for publication February 28, 2003, and accepted in revised form July 29, 2003.

Address correspondence to: Raif S. Geha, Enders 8, Division of Immunology, Children's Hospital, 300 Longwood Avenue, Boston, Massachusetts 02115, USA. Phone: (617) 355-7602; Fax: (617) 739-3145; E-mail: raif.geha@tch.harvard.edu.

Conflict of interest: The authors have declared that no conflict of interest exists.

Nonstandard abbreviations used: atopic dermatitis (AD); epicutaneous (EC); T cell receptor (TCR); high-power field (HPF); major basic protein (MBP); macrophage inflammatory protein (MIP); IFN-inducible protein-10 (IP-10); monocyte

chemoattractant protein-1 (MCP-1).
Listeria monocytogenes, Toxoplasma gondii, Leishmania amazonensis, Leishmania major, Candida albicans, Trypanosoma cruzi, and Chlamydia trachomatis (10-18).

Like T cells, APCs can be polarized. In vitro experiments with monocyte-derived DCs have shown that the presence of IL-12 or IFN- $\gamma$ during activation of DCs primes them for high IL-12 production and Th1-driving capacity (DC1). In contrast, prostaglandin E2 and IL-4 prime DCs for low IL-12 production ability and Th2-driving capacity (DC2) (19). Different microbial compounds polarize the maturation of human DCs differently. Extracts from the helminth Schistosoma mansoni induce the development of DC2 cells. In contrast, toxin from the intracellular bacterium Bordetella pertussis induces the development of DC1 cells (20). Liver DCs produce IL-10 in the presence of allogeneic cells, and stimulation via CD40 results in secretion of high levels of IL-10 from DCs derived from Peyer patches but not from spleen (21, 22). IL-10-producing DCs have been shown to promote the development of Th2 cells in vitro $(21,23)$.

Allergic diseases are characterized by a Th2 response to antigens. The role of IL-10 in regulating Th2 responses and in allergic diseases is not well understood. Exogenous administration of IL-10 to Balb/C mice sensitized intraperitoneally with OVA, then challenged by antigen inhalation, results in reduced airway eosinophilia (24). The effect of disruption of the IL-10 gene in the same system of OVA immunization and challenge is subject to controversy. Two reports showed significantly reduced eosinophilic infiltration and mucus production in lungs of IL-10-/- mice, with decreased levels of IL- 5 and higher levels of IFN- $\gamma$, but normal levels of IL-4, in the bronchoalveolar lavage 
fluid $(25,26)$. Another report showed increased eosinophilic airway inflammation in IL-10-/- mice, with decreased IL- 5 but normal IL-4, IL-13, and IFN- $\gamma$ levels in bronchoalveolar lavage fluid (27). In the same study, the airway response to OVA challenge was comparable in IL-10-/- and WT mice, but in two other studies it was found to be decreased in IL-10 ${ }^{-/-}$mice $(26,28)$.

Atopic dermatitis (AD) is an inflammatory skin disease that frequently occurs in subjects with personal or family history of atopic disease. Mechanical injury to the skin by scratching is an important feature of AD and has been shown to induce local expression of IL-10 mRNA in human skin (29). We have developed a mouse model of allergic skin inflammation elicited by epicutaneous (EC) sensitization with $\operatorname{OVA}(30,31)$. This model displays many of the features of human AD, including elevated total and specific IgE, a dermatitis characterized by infiltration of $\mathrm{CD}^{+} \mathrm{T}$ cells and eosinophils in the dermis, and by increased local expression of mRNA for Th2 cytokines. We used this model to assess the role of IL-10 in allergic skin inflammation.

\section{Methods}

Mice and sensitization. IL-10-/- mice and control WT mice of the same C57BL/ 6 genetic background were obtained from The Jackson Laboratory (Bar Harbor, Maine, USA). IL-10 ${ }^{-/-}$mice on Balb/C background were a generous gift from Donna Rennick (DNAX, Palo Alto, California, USA). OVA $323-339^{-}$-specific and $\mathrm{I}_{-\mathrm{A}^{\mathrm{d}} \text {-restricted }}$ DO11.10 T cell receptor $-\alpha \beta$ (TCR- $\alpha \beta)$ transgenic mice on Balb/C background were obtained from The Jackson Laboratory. Control Balb/C mice were from Taconics (Germantown, New York, USA). All mice were kept in a pathogen-free environment. All procedures performed on the mice were in accordance with the Animal Care and Use Committee of the Children's Hospital.

EC sensitization of 4- to 6-week-old female mice was performed as described previously (30). Briefly, the skin of anesthetized mice was shaved and tape stripped six times by transparent i.v. dressing (Tegaderm; Owens \& Minor Inc., Franklin, Massachusetts, USA). One hundred micrograms of OVA (Grade V; Sigma-Aldrich, St. Louis, Missouri, USA) in $100 \mu \mathrm{l}$ of normal saline, or placebo $(100 \mu \mathrm{l}$ of normal saline), was placed on a patch of sterile gauze $(1 \times 1 \mathrm{~cm})$, which was secured to the skin with a transparent bio-occlusive dressing. Each mouse had a total of three 1-week exposures to the patch separated from each other by 2 -week intervals. For intraperitoneal immunization, mice were injected at day 0 and 14 with $50 \mu \mathrm{g}$ OVA in alum and killed on day 28.

Histological analysis. Specimens obtained from patched areas on the skin 24 hours after the patch from the third sensitization were fixed in $10 \%$ buffered formalin and embedded in paraffin. Multiple $4-\mu \mathrm{m}$ sections were stained with H\&E. Individual inflammatory cell types were counted blinded in 15-20 high-power fields (HPFs) at $\times 1,000$.

Skin major basic protein by immunobistological analysis. Skin sections were embedded in Tissue-Tek oxacal- citriol (OCT) compound (Miles Laboratories Inc., Elkhart, Indiana, USA) on dry ice. Sections of $4 \mu \mathrm{m}$ were stained by an avidin-biotin method as described previously (32). The rabbit anti-major basic protein (anti-MBP) antibody was a kind gift from James Lee (Mayo Clinic, Scottsdale, Arizona, USA) (33).

$R N A$ preparation and PCR amplification of reverse-transcribed cDNA (RT-PCR). Skin biopsies were obtained at the end of the third sensitization and were immediately frozen in dry ice. The samples were homogenized in Trizol (GIBCO BRL, Life Technologies Grand Island, New York, USA) using a Polytron RT-3000 (Kinematica AG, Littau, Switzerland). RNA extraction was performed following the manufacturer's instruction. cDNA was synthesized from $10 \mu \mathrm{g}$ of total RNA in a $40-\mu \mathrm{l}$ reaction mix using Superscript II (GIBCO BRL). The primers used to amplify cDNA for $\beta_{2} \mathrm{~m}$, IL-10, IL-4, IL-5, and IFN- $\gamma$, and DNA amplification were as described previously (30). To quantify mRNA, a fixed amount of reverse-transcribed cellular mRNA was coamplified in the presence of serial dilutions of a multispecific internal plasmid control, pMUS3 (34), which contains nucleotide sequences of multiple cytokines. Quantification of cytokine mRNA was performed as previously described (31), and results were expressed as a ratio of cytokine cDNA to $\beta_{2} \mathrm{~m} \mathrm{cDNA}$.

$R$ Nase protection assay. Multiprobe template mCK-5 (BD Pharmingen, San Jose, California, USA) containing DNA templates for the chemokines lymphotactin, RANTES, eotaxin, macrophage inflammatory protein-1 (MIP-1), MIP-2, MIP-1 $\beta$, IFN-inducible protein-10 (IP-10), monocyte chemoattractant protein-1 (MCP-1), and TCA-3, and for the housekeeping genes L32 (ribosomal RNA) and GAPDH, was used. The assay was performed according to the manufacturer's protocol. The DNA templates were labeled with [32P]UTP $(3,000$ $\mathrm{Ci} / \mathrm{mmol}, 10 \mu \mathrm{Ci} / \mu \mathrm{l}$; PerkinElmer Life Sciences, Boston, Massachusetts, USA) and were hybridized overnight with $20 \mu \mathrm{g}$ of total RNA (from pooled samples of six mice in each group), followed by digestion with RNase A and $\mathrm{T} 1$.

In vitro IL-4 and IFN- $\gamma$ synthesis. Single-cell suspensions of spleen cells were prepared in complete RPMI 1640 (JRH Biosciences Inc., Lenexa, Kansas, USA) supplemented by $10 \%$ FCS, $1 \mathrm{mM}$ sodium pyruvate, $2 \mathrm{mM}$ L-glutamine, $0.05 \mathrm{mM}$ 2-ME, $100 \mathrm{U} / \mathrm{ml}$ penicillin, and $100 \mu \mathrm{g} / \mathrm{ml}$ streptomycin. Cells were cultured in the above medium at $2 \times 10^{6} / \mathrm{ml}$ in 24 -well plates in the presence of OVA $(50 \mu \mathrm{g} / \mathrm{ml})$ or in plates coated with anti-CD $3 \varepsilon \mathrm{mAb}(1 \mu \mathrm{g} / \mathrm{ml}$; Pharmingen). Supernatants were collected after 96 hours of culture, centrifuged, and frozen until use. IL- 4 and IFN- $\gamma$ in supernatants were determined by ELISA following the manufacturer's instructions (Pharmingen).

Preparation and in vitro priming of naive T cells from OVAspecific TCR transgenic mice. T cells from spleens of OVA transgenic mice were purified over mouse $T$ cell enrichment columns (R\&D Systems Inc., Minneapolis, Minnesota, USA) and consisted of $90-95 \% \mathrm{CD}^{+}$cells. APCs consisted of irradiated (28 Gy) splenocytes and 

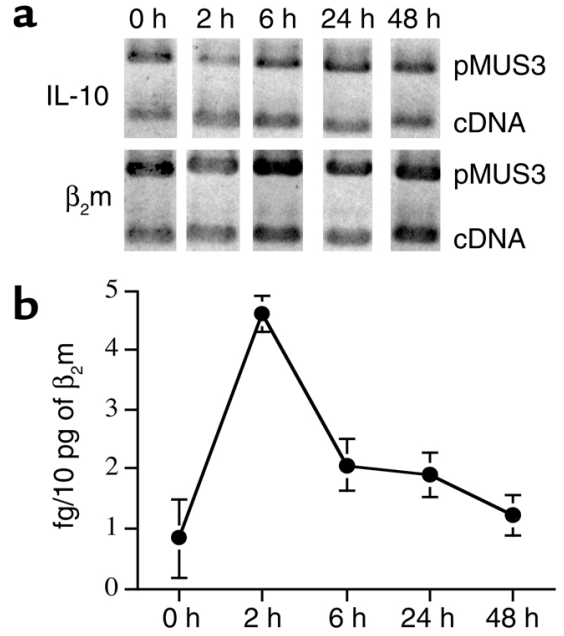

Figure 1

IL-10 mRNA expression in skin of C57BL/ 6 mice following tape stripping six times. (a) Results of a representative experiment. pMUS plasmid and cDNA were coamplified by Taq polymerase generating 300-bp PCR fragments from the pMUS plasmid and 252-bp fragments from IL-10 cDNA. In parallel, pMUS plasmid and cDNA samples were coamplified for $\beta_{2} m$ cDNA. The samples were run on $1.6 \%$ agarose gel and stained with ethidium bromide. (b) Pooled results of experiments using six mice. Cytokine transcripts were quantitated by RT-PCR and expressed as fg per 10 pg of $\beta_{2}$ m mRNA. Columns and error bars represent mean \pm SEM.

were pulsed for 1 hour at $37^{\circ} \mathrm{C}$ with $0.3 \mu \mathrm{M}$ of $\mathrm{OVA}_{323-339}$ peptide (Genosys, The Woodlands, Texas, USA). T cells were cultured in 24 -well plates $\left(10^{6}\right.$ cells per well) in the absence or presence of APCs $\left(5 \times 10^{6}\right.$ cells per well) obtained from either WT Balb/C mice or IL-10-/- mice on the Balb/C background. Cells recovered from 5-day cultures were washed twice, rested for another 5 days, then restimulated with irradiated APCs pulsed with OVA peptide. Supernatants were harvested after 24 hours and assayed for IL- 4 and IFN- $\gamma$ content.

$D C$ generation and in vivo transfer experiments. DCs were generated from spleens of IL-10-/- mice and genetically matched C57BL/ 6 controls. Eight to 12 spleens were cut into small fragments. Fragments were then digested with collagenase D $(1 \mathrm{mg} / \mathrm{ml}$; Worthington Biochemical Corp., Freehold, New Jersey, USA) for 25 minutes at room temperature and then treated with $0.1 \mathrm{M}$ EDTA ( $\mathrm{pH}$ 7.2) for 5 minutes. Undigested fibrous material was removed. Cells were recovered from the digest and washed. Light-density cells were selected by centrifugation in metrizamide medium $\left(1.068 \mathrm{~g} / \mathrm{cm}^{3}\right.$ $\mathrm{pH}$ 7.2; Sigma Chemical Co.). DCs were then further enriched by purification on MACS CD11c (N418) MicroBeads (Miltenyi Biotec, Auburn, California, USA), and purity was greater than $90 \% \mathrm{CD}_{11} \mathrm{c}^{+}$as determined by FACS.

For in vivo transfer experiments, DCs were pulsed with $50 \mu \mathrm{g} / \mathrm{ml}$ OVA for 18 hours and injected i.v. into C57BL $/ 6$ mice $\left(5 \times 10^{5}\right.$ cells per mouse $)$. After 10 days, splenocytes from mice that received DCs $\left(2 \times 10^{6} / \mathrm{ml}\right)$ were cultured in complete RPMI 1640 (JRH Biosciences
Inc.) supplemented with $1 \mathrm{mM}$ sodium pyruvate, $2 \mathrm{mM}$ L-glutamine, $0.05 \mathrm{mM}$ 2-ME, $100 \mathrm{U} / \mathrm{ml}$ penicillin, 100 $\mu \mathrm{g} / \mathrm{ml}$ streptomycin, and 3\% normal mouse serum (Cedarlane Laboratories Ltd., Hornby, Ontario, Canada) and stimulated with OVA $(50 \mu \mathrm{g} / \mathrm{ml})$. Supernatants were collected after 96 hours of culture and assayed for IL-4 and IFN- $\gamma$. In a parallel set of experiments, the skin of mice that received DCs was shaved, tape stripped, and challenged with OVA $(100 \mu \mathrm{g}$ in $100 \mu \mathrm{l}$ of normal saline) or placebo ( $100 \mu \mathrm{l}$ of normal saline), and placed on a patch of sterile gauze. Three days later, the skin was examined histologically and for expression of cytokines as described above.

Statistical analysis. A nonparametric Mann-Whitney $U$ test was used to compare the different mouse groups, since standard deviations varied widely between groups. A $P$ value smaller than 0.05 was considered statistically significant.

\section{Results}

Tape stripping induces rapid expression of IL-10 mRNA in normal mouse skin. Tape stripping of shaved skin is essential for EC sensitization, since antigen application to depilated, but not tape-stripped, skin or to skin of hairless mice, does not result in successful sensitization (data not shown). We used a competitive RT-PCR assay to examine the effect of tape stripping on IL-10 mRNA expression in mouse skin. Low levels of IL-10 mRNA were detectable in uninjured skin. After shaved skin was tape stripped six times, IL-10 mRNA expression rapidly increased, with peak levels attained within 2 hours after injury and returning to normal 48 hours later (Figure 1).

Eosinophil infiltration is decreased in OVA-sensitized skin sites of $I L-10^{-/-}$mice. Dermal infiltration with eosinophils is an important feature of our model of allergic skin inflammation elicited by EC allergen application (30, 31). The rapid increase of IL-10 mRNA expression following tape stripping prompted us to examine the role of IL-10 in allergic skin inflammation induced by EC sensitization of tape-stripped skin. We compared the dermal skin infiltrate in the skin of EC-sensitized
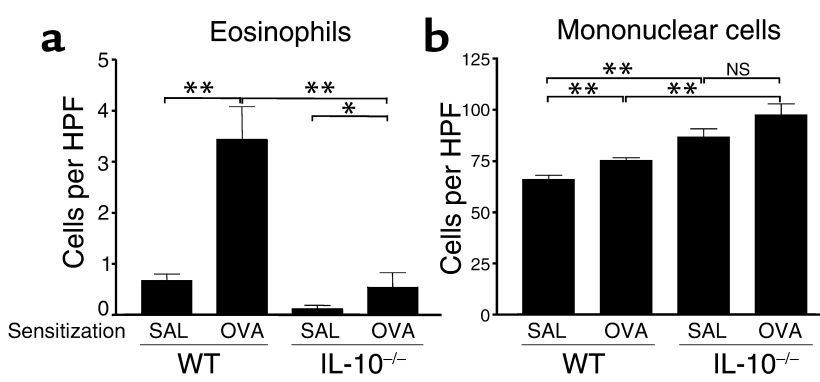

\section{Figure 2}

Number of infiltrating eosinophils (a) and mononuclear cells (b) in OVA- and saline-sensitized (SAL) skin sites of IL-10-/- mice and WT controls. The columns and error bars represent mean \pm SEM per HPF of cells calculated by examining $15-20 \mathrm{HPFs}$ per mouse $(n=6)$. ${ }^{*} P \leq 0.05 ;{ }^{*} P \leq 0.01$. 
IL-10-/- mice and C57BL/6 WT controls. There was no difference in the numbers of eosinophils in the noninjured skin of IL-10-/- mice $(0.105+0.03$ per HPF, compared with $0.125+0.04$ per HPF in WT skin). Salinesensitized skin sites from IL-10-/- mice and WT controls contained only a small number of eosinophils (Figure 2 , $a$ and $b$ ). OVA sensitization caused a vigorous and significant increase in the number of eosinophils in the skin of genetically matched C57BL/6 mice, consistent with previous observations that our model is operative in this strain (31). In contrast, there was only a modest increase in the number of eosinophils in the skin of IL-10-/- mice. Furthermore, there were significantly fewer eosinophils in OVA-sensitized skin of IL-10-/mice compared with WT controls (Figure 2, a and b). OVA sensitization caused an increase in mononuclear cell infiltration in both WT and IL-10/- mice. Both saline- and OVA-sensitized sites from IL-10 ${ }^{-/-}$mice had significantly more mononuclear cells than similarly sensitized sites from WT controls (Figure 2, a and b).

The decreased numbers of eosinophils in the skin of IL-10 $/-$ mice could have resulted either from their failure to infiltrate the skin or from impaired survival. The eosinophil product myelin basic protein (MBP) has been used as a marker for eosinophils in tissues and remains detectable following eosinophil apoptosis (35). MBP staining was readily detectable in saline-treated skin sites and markedly increased following OVA sensitization in WT mice. In contrast, little MBP staining was detectable in either saline-treated or OVA-sensitized skin sites of IL-10-/- mice (Figure 3a). These results suggest that IL-10 is critical for eosinophil infiltration of the skin following EC sensitization with antigen.

IL-5 and eotaxin expression is decreased in OVA-sensitized skin sites of $I L-10^{-/-}$mice. We have previously shown that expression of the eosinophil chemoattractant eotaxin is upregulated in OVA-sensitized skin sites (31), and that the eotaxin receptor CCR3 is critical for eosinophil infiltration in the skin in our model (36). We compared expression of mRNA for eotaxin in IL-10-/- mice and WT controls, using an RNase protection assay that detects mRNA for nine chemokines. Expression of MIP-2, and, to a lesser extent, IP-10 and MIP-1 $\beta$, was increased in saline-sensitized skin from IL-10-/- mice compared with WT controls. As we have previously shown in Balb/C mice (31), there was an increased expression of eotaxin and of several other chemokines in OVA-sensitized skin of WT C57BL/6 mice that included MIP-1 $\alpha$, IP-10, MCP-1, and TCA-3. In contrast, OVA-sensitized skin sites from IL-10/-- mice

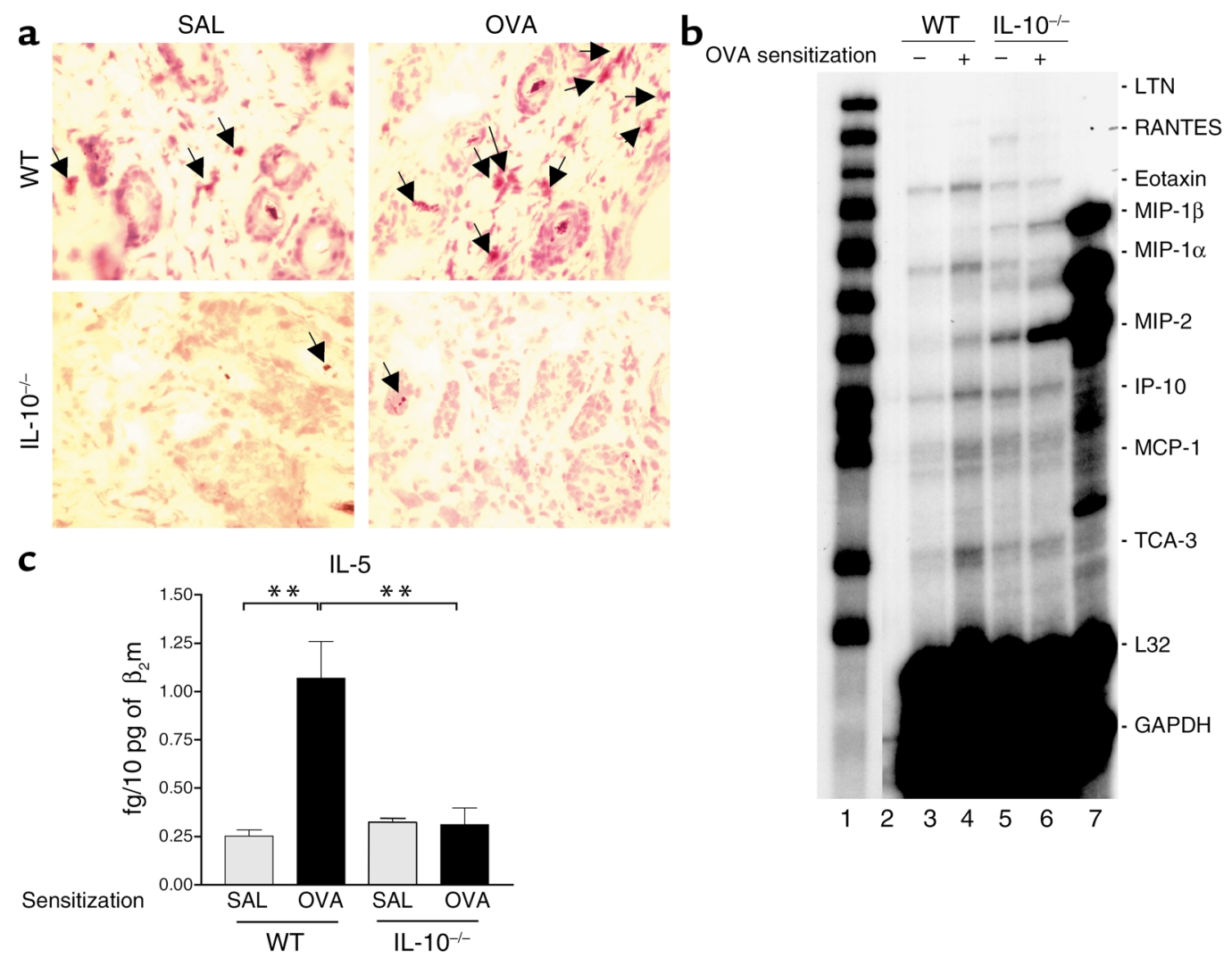

Figure 3

(a) Immunoperoxidase staining of sensitized skin sites for MBP. MBP stains brown. Arrows indicate MBP deposits. (b) RNase protection assay for chemokine expression in pooled total mRNA of WT and IL-10-/- mouse groups (lanes 3-6). Lane 1 represents the labeled probe, lane 2 represents the yeast tRNA negative control, and lane 7 represents total mRNA from CD3-stimulated mouse splenocytes (positive control). Results represent two independent experiments. (c) IL-5 mRNA expression in sensitized skin sites. Pooled results of experiments using six OVA-sensitized mice and six saline-sensitized controls. Columns and error bars represent mean $\pm \mathrm{SEM}$. ${ }^{*} P \leq 0.01$. 

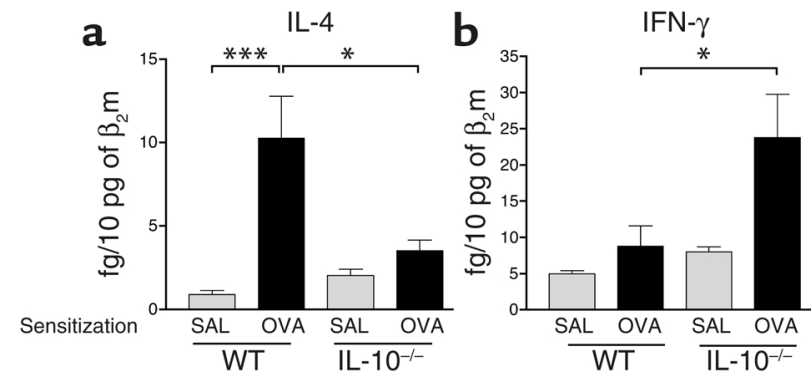

Figure 4

IL-4 (a) and IFN- $\gamma(\mathbf{b})$ expression in sensitized skin of IL-10-/- mice and WT controls. Levels were normalized to $\beta_{2} \mathrm{~m}$. Pooled results of experiments using six OVA-sensitized mice and six saline-sensitized controls. Bars represent mean \pm SEM. ${ }^{*} P \leq 0.05 ;{ }^{* *} P \leq 0.001$.

showed no detectable increase in the expression of these chemokines. Expression of MIP-2, and, to a lesser extent, MIP-1 $\beta$, increased in OVA-sensitized skin from $\mathrm{IL}-10^{-/-}$mice. The impaired expression of eotaxin in the OVA-sensitized skin of IL-10 $0^{-/-}$mice may contribute to the impaired accumulation of eosinophils following EC sensitization.

IL-5 is important for skin eosinophilia in our model (31). As we have previously shown (30), there was a significant increase in skin IL-5 mRNA in WT mice following OVA sensitization. In contrast, IL-5 mRNA did not significantly increase in OVA-sensitized skin of IL-10 $-1-$ mice (Figure $3 \mathrm{c}$ ). These results suggest that IL-10 plays an important role in IL-5 expression at EC-sensitized skin sites.

IL-4 expression is decreased and IFN- $\gamma$ expression is increased in EC-sensitized skin sites of IL-10-/- mice. Since skin eotaxin expression in our model is dependent on IL-4 (31), and since IL- 5 is produced by Th 2 cells, we examined the expression of the prototypic Th2 and Th1 cytokines IL- 4 and IFN- $\gamma$ in the skin of IL-10-/- mice and WT controls. Low and comparable levels of IL-4 and IFN- $\gamma$ mRNA were detected in salinesensitized skin from both WT and IL-10-/mice (Figure 4). Consistent with previous results (30), expression of IL-4 mRNA, but not IFN- $\gamma$ mRNA, markedly increased in OVA-sensitized skin sites of WT mice. In contrast, IL-4 mRNA did not significantly increase in OVA-sensitized skin of IL-10 ${ }^{-/-}$ mice, whereas IFN- $\gamma$ mRNA was significantly increased. These results suggest that following EC sensitization, IL-10 normally

\section{Figure 5}

Cytokine production by spleen cells from IL-10 $10^{-/-}$mice and WT controls. Splenocytes were stimulated in vitro for 4 days with OVA (a) or anti-CD3 mAb (CD3) (b). IL-4 and IFN- $\gamma$ levels in the supernatants were assayed by ELISA. Results are pooled from experiments using six mice in each group. Columns and error bars represent mean \pm SEM. ${ }^{*} P \leq 0.05 ;{ }^{*} P \leq 0.01$. a
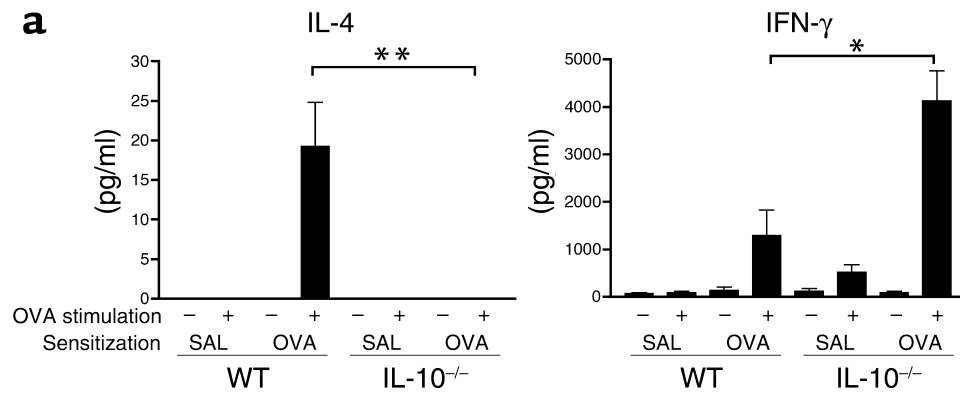

b
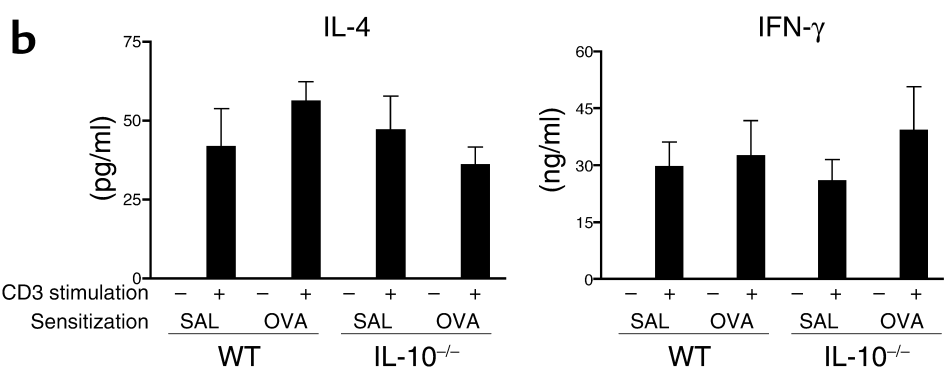

skews the cytokine profile of infiltrating $T$ cells towards Th2 and away from Th1.

IL-10-/- mice have a decreased systemic Th2 response and an increased systemic Th1 response to EC sensitization. The abnormal Th cytokine profile in OVA-sensitized skin sites of IL-10-/- mice prompted us to examine the systemic Th response of these mice to EC sensitization. As we have previously described (36), splenocytes from WT mice EC sensitized with OVA, but not with saline, secreted IL-4 and IFN- $\gamma$ following OVA stimulation in vitro. In contrast, splenocytes from IL-10-/- mice EC sensitized with OVA secreted no measurable amounts of IL-4, but significantly more IFN- $\gamma$ than splenocytes of WT controls (Figure 5a). This abnormal profile of cytokine secretion was specific to the immunizing antigen, since splenocytes from IL-10-/- mice and WT controls secreted equivalent amounts of IL- 4 and IFN- $\gamma$ following stimulation with anti-CD3 $\mathrm{mAb}$ (Figure $5 \mathrm{~b}$ ). These results suggest that IL-10 plays an important role in the development of the systemic Th2 response to EC-introduced antigen.

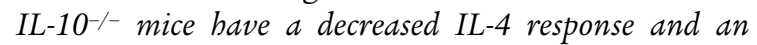
increased IFN- $\gamma$ response to intraperitoneal immunization. We next investigated whether the skewed Th1 response of IL-10-/- mice is restricted to the EC route of immunization. Figure 6 shows that splenocytes from intraperitoneally immunized IL-10-/- mice secreted significantly less IL-4, and significantly more IFN- $\gamma$, than splenocytes of similarly immunized WT controls. These results suggest that IL-10 promotes skewing the systemic Th response towards Th2, and inhibits the development of a systemic Th1 response, regardless of the route of antigen immunization.

IL-10-deficient APCs skew the cytokine profile of antigen-specific $\mathrm{T}$ cells to a Th1 response in vitro. IL-10 is expressed in both APCs and T cells (1). The skewing of the cytokine response of $\mathrm{T}$ cells to antigen 

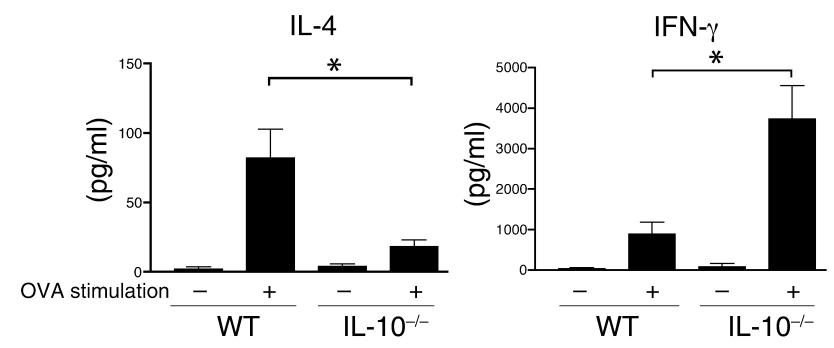

Figure 6

Cytokine production by spleen cells from IL-10/- mice and WT controls immunized intraperitoneally with OVA. Assays were performed as described in the legend to Figure 5. Results are pooled from experiments using six mice in each group. Columns and error bars represent mean \pm SEM. ${ }^{*} P \leq 0.05$.

towards Th1 in IL-10-/- mice could be due to IL-10 deficiency in the APC, the T cell, or both. To determine the role of the APC in skewing the response of $\mathrm{IL}-10^{-/-}$mice towards Th1, we examined the cytokine response of purified T cells from TCR OVA transgenic mice to their cognate peptide presented by APCs from either IL-10-/or WT mice on the same Balb/C background. Figure 7 shows that APCs from IL-10/- mice elicited significantly less IL-4 secretion than APCs from WT mice. In contrast, they elicited significantly more IFN- $\gamma$ secretion than WT APCs did. These results suggest that the regulatory effect of IL-10 on Th development is exerted, at least in part, at the level of the APC.

Both DC-derived and T cell-derived IL-10 cells promote the $T h 2$ response and inhibit the Th1 response to antigen in vivo. We next investigated the contribution of IL-10 derived from DCs and T cells on the Th cell response to OVA. DCs purified from WT and IL-10 $10^{--}$mice were pulsed with OVA for 18 hours, then injected i.v. into WT and IL-10 $1 /-$ mice. Ten days later, we examined the ability of splenocytes from these mice to secrete IL- 4 and IFN- $\gamma$ in response to OVA stimulation. Figure 8a shows the results of IL-4 secretion. As expected, T cells from IL-10 $10^{-/}$mice immunized with IL-10-/- DCs secreted significantly less IL-4 than T cells from WT mice immunized with WT DCs. T cells from WT mice immunized with IL-10-/- DCs also secreted significantly less IL-4 than $T$ cells from WT mice immunized with WT DCs (Figure 8a, left panel). Immunization of IL-10-/- mice with WT DCs partially corrected their impaired IL-4 secretion (Figure 8a, right panel). These results suggest that DCs are important for IL-10 promotion of the $\mathrm{Th} 2$ response in vivo.

Comparison of the response of WT and IL-10-/- mice to $\mathrm{DC}$ immunization revealed that $\mathrm{T}$ cells from $\mathrm{IL}-10^{-/-}$ mice immunized with WT DCs produced significantly less IL-4 than T cells from WT controls immunized with WT DCs (Figure 8a, upper row). This result, together with the incomplete correction of the IL-4 response of IL-10-/- mice by WT DCs, suggests that T cells also play a role in IL-10 promotion of the Th2 response to antigen. Comparison of IL-4 secretion by $\mathrm{T}$ cells from WT and $\mathrm{IL}-10^{-/-}$mice immunized with
IL-10-/- DCs revealed that both responses were equivalently low (Figure 8a, lower row), suggesting that DCs exert a dominant effect on Th2 skewing by IL-10.

Figure $8 \mathrm{~b}$ shows the results of IFN- $\gamma$ secretion. Immunization of WT mice with either WT or IL-10-/- DCs resulted in no detectable IFN- $\gamma$ production (Figure $8 \mathrm{~b}$, left panel). In contrast, splenocytes from IL- $10^{-/-}$mice produced IFN- $\gamma$, with more being produced in animals immunized with IL-10-/- DCs, than in animals immunized with WT DCs (Figure 8b, right column). These results suggest that both DC-derived and T cells play a role in IL-10 downregulation of the Th1 response.

Role of DC-derived IL-10 in allergic skin inflammation. To determine the role of DC-derived IL-10 in allergic skin inflammation, we performed an antigen challenge to the skin of WT recipient mice that were immunized with OVA-pulsed DCs derived from IL-10-/- mice or WT controls. Three days later, the skin was examined histologically, and expression of cytokines was measured by RT-PCR. Figure 9a shows that OVA challenge of skin from mice immunized with OVA-pulsed WT DCs resulted in significant eosinophil and mononuclear cell infiltration compared with saline challenge of skin from the same mice. There was no significant eosinophil infiltration in OVA-challenged skin of mice immunized with OVA-pulsed IL-10-deficient DCs, although mononuclear cell infiltration was intact. Figure $9 \mathrm{~b}$ shows that IL-4, but not IFN- $\gamma$, expression significantly increased in OVA-challenged skin from mice immunized with OVA-pulsed WT DCs. In contrast, IL-4 expression did not significantly increase, whereas IFN- $\gamma$ expression significantly increased in OVA-challenged skin from mice immunized with OVA-pulsed IL-10-/- DCs. These results suggest that IL-10 derived from DCs plays an important role in Th2mediated allergic skin sensitization.

\section{Discussion}

The results of this study suggest that IL-10 promotes the development of a Th2 response to antigen and is essential for skin infiltration with eosinophils in a mouse model of allergic dermatitis.

IL-10 can be expressed by both keratinocytes and cells of hemopoietic origin that are present in the skin,

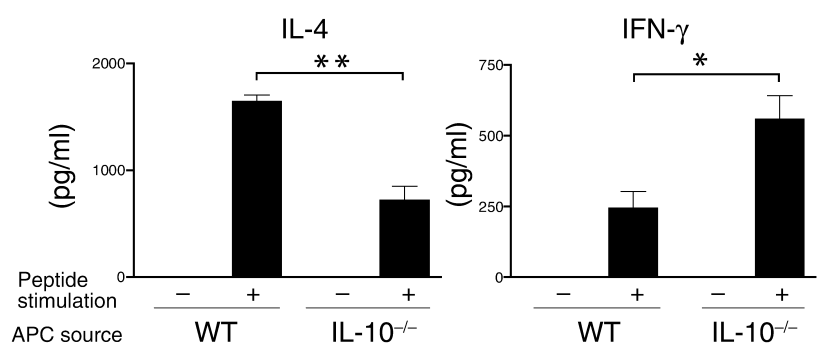

Figure 7

Cytokine production by splenic T cells from TCR OVA transgenic mice primed and then restimulated in vitro with APCs pulsed with $\mathrm{OVA}_{323-339}$ peptide that was derived from IL-10-/- mice or WT controls. Columns and error bars represent mean \pm SEM of three experiments. ${ }^{*} P \leq 0.05 ;{ }^{*} P \leq 0.01$. 


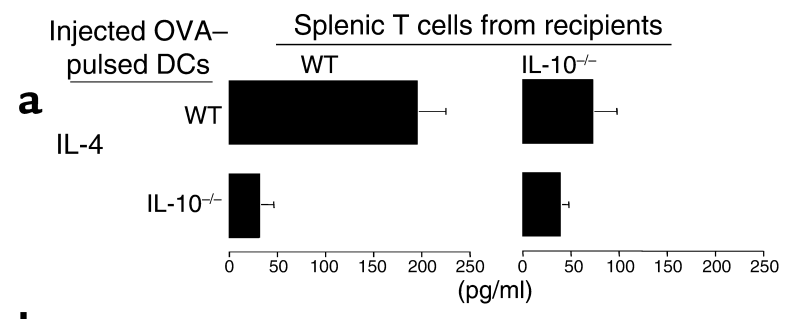

b

IFN- $\gamma$

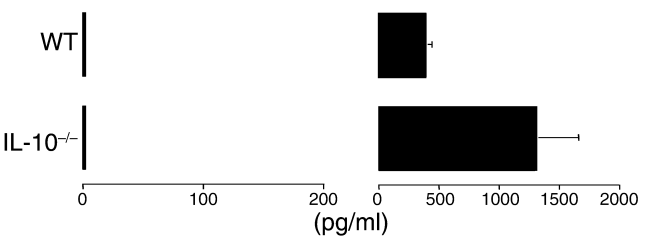

\section{Figure 8}

In vitro IL-4 (a) and IFN- $\gamma(\mathbf{b})$ production in response to OVA stimulation by splenocytes from IL-10-/- mice and WT controls immunized i.v. with OVA-pulsed DCs purified from IL-10/- or WT mice. Pooled results of experiments using four mice in each group. Blocks and error bars represent mean \pm SEM of three experiments. Spleen cells from animals immunized with unpulsed DCs secreted no detectable IL-4 or IFN- $\gamma$ in response to OVA stimulation (data not shown).

including DCs, macrophages, mast cells, and lymphocytes $(2,37)$. Our investigation was prompted by the observation that IL-10 mRNA is rapidly expressed in mouse skin following mechanical injury by tape stripping (Figure 1). This result is consistent with previous findings in human skin subjected to tape stripping (29). Dermal infiltration with eosinophils is an important feature of $\mathrm{AD}$ and a hallmark of allergic skin inflammation induced by EC sensitization with OVA (30). Skin from $\mathrm{IL}-10^{-/}$mice failed to exhibit eosinophil infiltration following EC sensitization with OVA (Figure 2a). This is not due to poor survival of eosinophils, since there was virtually no detectable staining for the eosinophil product MBP in OVA-sensitized skin of IL-10-/- mice (Figure 3a). Mononuclear cell recruitment to both saline and OVA sensitized in the skin of IL-10-/- mice was slightly increased (Figure $2 \mathrm{~b})$. This is consistent with previous observations that deletion of IL-10 results in increased infiltration of mononuclear cells into tissues $(38,39)$ and with our observation of increased expression of MIP- $1 \beta$ and MIP-2 in the skin of IL-10-/- mice (Figure $3 \mathrm{~b}$ ). Expression of these two chemokines has been shown to be downregulated by IL-10 (40-44).

We have previously shown that expression of IL-5, IL-4, and eotaxin mRNA is increased in OVA-sensitized skin sites, that eotaxin expression is dependent on IL-4, and that eosinophil infiltration is dependent on IL-4, IL-5, and the eotaxin ligand CCR3 (31). IL-10/-- mice failed to increase the expression of eotaxin, IL-4, and IL-5 mRNA in their skin following EC sensitization with OVA (Figure 3, b and c; and Figure 4). More importantly, IL-10-/ mice had a diminished Th2 and an increased Th1 systemic response to EC and intraperitoneal sensitization. Splenocytes from these mice were impaired in their ability to secrete IL-4 in response to OVA (Figures
5 and 6). In contrast, their ability to secrete IFN- $\gamma$ was significantly enhanced. These results are consistent with the previously observed decreased IL-5 production by splenocytes of IL-10 $-/-$ mice immunized intraperitoneally with antigen (25). Taken together, our results suggest that IL-10 promotes the development of Th2 cytokines, and inhibits the development of Th1 cytokines in response to antigen. Previous observations have shown that administration of exogenous IL-10 inhibits both Th1 and Th2 responses to antigen (45-47). The apparent contradictory effects of administration of exogenous IL-10 (dampening of both Th1 and Th2 responses) versus ablation of the IL-10 gene (decreased Th2 response and increased Th1 response) suggest that IL-10 plays different roles in the induction of Th responses, versus the regulation of established Th responses.

Our data suggest that both APCs and T cells play a role in IL-10 promotion of the Th2 response to antigen. In the presence of the cognate peptide, WT APCs induced significantly more IL-4 secretion and significantly less IFN- $\gamma$ secretion by OVA TCR transgenic $\mathrm{T}$ cells, than IL-10-/- APCs (Figure 7). This is consistent with the observation that Th2 differentiation is induced by DCs from Peyer patches that produce IL-10, but not by APCs from spleen that fail to produce IL-10 (21). Overnight incubation of IL-10-/- APCs with IL-10 or addition of IL-10 to cultures containing IL-10-deficient APCs and TCR OVA transgenic T cells failed to correct the skewing towards Th1 cytokine production induced by IL-10-- APCs (data not shown). This suggests that IL-10 deficiency affects APCs irreversibly in vivo, or that the conditions of exposure to IL-10 in vitro (i.e., timing, dose, presence of other cytokines) did not replicate those in vivo.
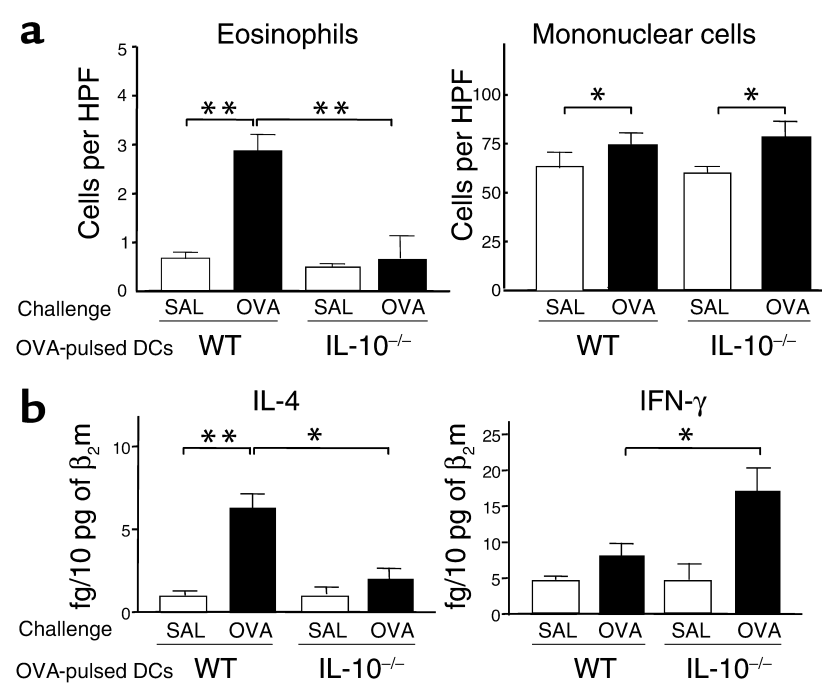

Figure 9

Number of infiltrating eosinophils and mononuclear cells (a), and cytokine expression in OVA- and saline-sensitized skin sites of WT mice immunized i.v. with OVA-pulsed DCs derived from IL-10-- or WT mice (b). The columns and error bars represent mean $\pm \mathrm{SE}(n=6$ per group). ${ }^{*} P \leq 0.05 ;{ }^{*} P \leq 0.01$. 
The role of APCs in IL-10 skewing the Th response towards Th2 was further confirmed by the results of in vivo immunization experiments using antigenpulsed DCs. Antigen-pulsed WT DCs elicited a significantly higher IL-4 response to OVA in T cells from both WT and IL-10/- mice compared with antigenpulsed IL-10-/- DCs. Conversely, antigen-pulsed WT DCs elicited a significantly lower IFN- $\gamma$ response in IL-10-/- mice than antigen-pulsed IL-10-/- DCs (Figure 8). Furthermore, we were able to demonstrate that DC-derived IL-10 plays an important role in allergic skin inflammation. Eosinophil infiltration and expression of the Th2 cytokine IL-4 were significantly decreased, whereas expression of the Th1 cytokine INF- $\gamma$ was significantly increased in OVA-challenged skin of WT mice immunized with OVA-pulsed IL-10deficient DCs, compared with WT mice immunized with OVA-pulsed WT DCs. The fact that we observed these differences suggests that cross-presentation of antigens by recipient APCs is not likely to have played a major role in our experiments. Taken together, these results suggest that APCs play an important role in IL-10 promotion of the in vivo response to antigen towards Th2.

A role for $\mathrm{T}$ cells in IL-10 promotion of the Th2 response to antigen was suggested by the observation that $\mathrm{T}$ cells from WT mice secreted significantly higher amounts of IL-4 than T cells from IL-10-/mice in response to immunization with antigenpulsed WT DCs. Conversely, T cells from IL-10-/mice, but not from WT mice, secreted IFN- $\gamma$ in response to immunization with antigen-pulsed WT DCs. The fact that IL-4 secretion by T cells from WT and IL-10 $1 /-$ mice immunized with IL-10 $1 /$ - DCs made equivalently poor IL-4 responses suggests that DCs may play a more dominant role than T cells in IL-10 promotion of a Th2 response.

A likely target of IL-10 in the regulation of the Th response is the APC itself. IL-10 has been shown to downregulate IL-12 production by DCs $(48,49)$. IL-12 is critical for the development of Th1 cells and their production of IFN- $\gamma$, which in turn downregulates Th2 cytokine production. Decreased IL-4 production may amplify the effect of IL-10 deficiency on the Th response, since IL-4 treatment causes DCs to prime naive $\mathrm{CD}^{+} \mathrm{T}$ cells to secrete IL-4 (50). Taken together with our findings, these observations suggest that DCs may be both a source and a target for IL-10 in the skewing of the Th response towards Th2.

The hygiene hypothesis is based on the observation that the incidence of allergic diseases rises with improvement in hygiene. Because there is also a concomitant rise in the incidence of autoimmune diseases, it has been suggested that production of IL-10 by regulatory $\mathrm{T}$ cells following infections suppresses both Th1- and Th2-mediated immune responses (51). Our results clearly show that IL-10 promotes Th2 responses and suppresses Th1 responses in two models of allergic sensitization that respectively used the EC route and the intraperitoneal route of immunization. These findings support a major importance of immune deviation from Th1 to Th2 responses rather than that of reduced immune suppression by regulatory cells and cytokines in explaining the increased prevalence of allergy consequent to reduced microbial burden in childhood. Finally, EC sensitization of mice is directly relevant to human sensitization because it mimics allergen sensitization via abraded skin in patients with AD. Furthermore, IL-10 is overexpressed in the skin of $\mathrm{AD}$ patients (52). Interventions directed at downregulating IL-10 production in atopic individuals at the time of allergen exposure may provide a novel therapeutic modality for the prevention of allergic diseases.

\section{Acknowledgments}

We thank Donna Rennick for the IL-10-/- mice on Balb/C background, Emiko Mizoguchi for her help in MBP staining, and Ali Yalcindag for assistance. This work is supported by NIH grant AI-31541 and by the Baxter Corporation.

1. Moore, K.W., O'Garra, A., de Waal Malefyt, R., Vieira, P., and Mosmann, T.R. 1993. Interleukin-10. Annu. Rev. Immunol. 11:165-190.

2. Moore, K.W., de Waal Malefyt, R., Coffman, R.L., and O'Garra, A. 2001. Interleukin-10 and the interleukin-10 receptor. Annu. Rev. Immunol. 19:683-765.

3. de Waal Malefyt, R., Abrams, J., Bennett, B., Figdor, C.G., and de Vries, J.E. 1991. Interleukin 10(IL-10) inhibits cytokine synthesis by human monocytes: an autoregulatory role of IL-10 produced by monocytes. J. Exp. Med. 174:1209-1220.

4. Fiorentino, D.F., Zlotnik, A., Mossmann, T.R., Howard, M., and O'Garra, A. 1991. IL-10 inhibits cytokine production by activated macrophages. J. Immunol. 147:3815-3822.

5. de Waal Malefyt, R., et al. 1993. Effects of IL-13 on phenotype, cytokine production, and cytotoxic function of human monocytes. Comparison with IL-4 and modulation by IFN-gamma or IL-10. J. Immunol. 151:6370-6381.

6. D'Andrea, A., et al. 1993. Interleukin 10 (IL-10) inhibits human lymphocyte interferon gamma-production by suppressing natural killer cell stimulatory factor/IL-12 synthesis in accessory cells. J. Exp. Med. 178:1041-1048.

7. Akdis, C.A., Joss, A., Akdis, M., Faith, A., and Blaser, K. 2000. A molecular basis for T cell suppression by IL-10: CD28-associated IL-10 receptor inhibits CD28 tyrosine phosphorylation and phosphatidylinositol 3-kinase binding. FASEB J. 14:1666-1668.

8. Yssel, H., et al. 1992. IL-10 is produced by subsets of human CD4+ T cell clones and peripheral blood T cells. J. Immunol. 149:2378-2384.

9. Wagner, R.D., Maroushek, N.M., Brown, J.F., and Czuprynski, C.J. 1994. Treatment with anti-interleukin-10 monoclonal antibody enhances early resistance to but impairs complete clearance of Listeria monocytogenes infection in mice. Infect. Immun. 62:2345-2353.

10. Reed, S.G., et al. 1994. IL-10 mediates susceptibility to Trypanosoma cruzi infection. J. Immunol. 153:3135-3140.

11. Dai, W.J., Kohler, G., and Brombacher, F. 1997. Both innate and acquired immunity to Listeria monocytogenes infection are increased in IL-10deficient mice. J. Immunol. 158:2259-2267.

12. Neyer, L.E., et al. 1997. Role of interleukin-10 in regulation of T celldependent and $\mathrm{T}$ cell-independent mechanisms of resistance to Toxoplasma gondii. Infect. Immun. 65:1675-1682.

13. Vazquez-Torres, A., Jones-Carson, J., Wagner, R.D., Warner, T., and Balish, E. 1999. Early resistance of interleukin-10 knockout mice to acute systemic candidiasis. Infect. Immun. 67:670-674.

14. Yang, X., Gartner, J., Zhu, L., Wang, S., and Brunham, R.C. 1999. IL-10 gene knockout mice show enhanced Th1-like protective immunity and absent granuloma formation following Chlamydia trachomatis lung infection. J. Immunol. 162:1010-1017.

15. Igietseme, J.U., et al. 2000. Suppression of endogenous IL-10 gene expression in dendritic cells enhances antigen presentation for specific Th1 induction: potential for cellular vaccine development. J. Immunol. 164:4212-4219.

16. Kane, M.M., and Mosser, D.M. 2001. The role of IL-10 in promoting disease progression in leishmaniasis. J. Immunol. 166:1141-1147.

17. Murphy, M.L., Wille, U., Villegas, E.N., Hunter, C.A., and Farrell, J.P. 2001. IL-10 mediates susceptibility to Leishmania donovani infection. Eur. J. Immunol. 31:2848-2856. 
18. Jones, D.E., Ackermann, M.R., Wille, U., Hunter, C.A., and Scott, P. 2002. Early enhanced Th1 response after Leishmania amazonensis infection of C57BL/ 6 interleukin-10-deficient mice does not lead to resolution of infection. Infect. Immun. 70:2151-2158.

19. Sato, M., Iwakabe, K., Kimura, S., and Nishimura, T. 1999. Functional skewing of bone marrow-derived dendritic cells by Th1- or Th2-inducing cytokines. Immunol. Lett. 67:63-68.

20. de Jong, E.C., et al. 2002. Microbial compounds selectively induce Th1 cell-promoting or Th2 cell-promoting dendritic cells in vitro with diverse th cell-polarizing signals. J. Immunol. 168:1704-1709.

21. Iwasaki, A., and Kelsall, B.L. 1999. Freshly isolated Peyer patch, but not spleen, dendritic cells produce interleukin 10 and induce the differentiation of T helper type 2 cells. J. Exp. Med. 190:229-239.

22. Khanna, A., et al. 2000. Effects of liver-derived dendritic cell progenitors on Th1- and Th2-like cytokine responses in vitro and in vivo. J. Immunol. 164:1346-1354.

23. Palma, J.P., Yauch, R.L., Kang, H.K., Lee, H.G., and Kim, B.S. 2002. Preferential induction of IL-10 in APC correlates with a switch from Th1 to Th2 response following infection with a low pathogenic variant of Theiler's virus. J. Immunol. 168:4221-4230.

24. Zuany-Amorim, C., et al. 1995. Interleukin-10 inhibits antigen-induced cellular recruitment into the airways of sensitized mice. J. Clin. Invest. 95:2644-2651.

25. Yang, X., Wang, S., Fan, Y., and Han, X. 2000. IL-10 deficiency prevents IL-5 overproduction and eosinophilic inflammation in a murine model of asthma-like reaction. Eur. J. Immunol. 30:382-391.

26. Makela, M.J., et al. 2000. IL-10 is necessary for the expression of airway hyperresponsiveness but not pulmonary inflammation after allergic sensitization. Proc. Natl. Acad. Sci. U. S. A. 97:6007-6012.

27. Tournoy, K.G., Kips, J.C., and Pauwels, R.A. 2000. Endogenous interleukin-10 suppresses allergen-induced airway inflammation and nonspecific airway responsiveness. Clin. Exp. Allergy. 30:775-783.

28. Justice, J.P., et al. 2001. IL-10 gene knockout attenuates allergen-induced airway hyperresponsiveness in C57BL/6 mice. Am. J. Physiol. Lung Cell. Mol. Physiol. 280:L363-L368.

29. Nickoloff, B.J., and Naidu, Y. 1994. Perturbation of epidermal barrier function correlates with initiation of cytokine cascade in human skin. J. Am. Acad. Dermatol. 30:535-546.

30. Spergel, J., et al. 1998. Epicutaneous sensitization with protein antigen induces localized allergic dermatitis and hyperresponsiveness to metacholine after single exposure to aerosolized antigen in mice. J. Clin. Invest. 101:1614-1622.

31. Spergel, J.M., Mizoguchi, E., Oettgen, H., Bhan, A.K., and Geha, R.S. 1999. Roles of TH1 and TH2 cytokines in a murine model of allergic dermatitis. J. Clin. Invest. 103:1103-1111.

32. Mombaerts, P., et al. 1993. Spontaneous development of inflammatory bowel disease in T cell receptor mutant mice. Cell. 75:275-282.

33. Lee, J.J., et al. 1997. Interleukin-5 expression in the lung epithelium of transgenic mice leads to pulmonary changes pathognomonic of asthma. J. Exp. Med. 185:2143-2156.

34. Shire, D. 1993. An invitation to an open exchange of reagents and information useful for the measurements of cytokine mRNA levels by PCR. Eur. Cytokine Netw. 4:161-162.
35. Peters, M.S., Schroeter, A.L., and Gleich, G.J. 1983. Immunofluorescence identification of eosinophil granule major basic protein in the flame figures of Wells' syndrome. Br. J. Dermatol. 109:141-148.

36. Ma, W., et al. 2002. CCR3 is essential for skin eosinophilia and airway hyperresponsiveness in a murine model of allergic skin inflammation. J. Clin. Invest. 109:621-628. doi:10.1172/JCI200214097.

37. Enk, A.H., and Katz, S.I. 1992. Identification and induction of keratinocyte-derived IL-10. J. Immunol. 149:92-95.

38. Shanley, T.P., Vasi, N., and Denenberg, A. 2000. Regulation of chemokine expression by IL-10 in lung inflammation. Cytokine. 12:1054-1064.

39. Kawachi, S., et al. 2000. Cytokine and endothelial cell adhesion molecule expression in interleukin-10-deficient mice. Am. J. Physiol. Gastrointest. Liver Physiol. 278:G734-G743.

40. Kasama, T., Strieter, R.M., Lukacs, N.W., Burdick, M.D., and Kunkel, S.L. 1994. Regulation of neutrophil-derived chemokine expression by IL-10. J. Immunol. 152:3559-3569.

41. Driscoll, K.E., et al. 1998. Interleukin-10 regulates quartz-induced pulmonary inflammation in rats. Am. J. Physiol. 275:L887-L894.

42. Guo, H., et al. 1998. Regulation of beta-chemokine mRNA expression in adult rat astrocytes by lipopolysaccharide, proinflammatory and immunoregulatory cytokines. Scand. J. Immunol. 48:502-508.

43. Yoshidome, H., Kato, A., Edwards, M.J., and Lentsch, A.B. 1999. Interleukin-10 inhibits pulmonary NF-kappaB activation and lung injury induced by hepatic ischemia-reperfusion. Am. J. Physiol. 277:L919-L923.

44. Yoshidome, H., Kato, A., Edwards, M.J., and Lentsch, A.B. 1999. Interleukin-10 suppresses hepatic ischemia/reperfusion injury in mice: implications of a central role for nuclear factor kappaB. Hepatology. 30:203-208.

45. Li, L., Elliott, J.F., and Mosmann, T.R. 1994. IL-10 inhibits cytokine production, vascular leakage, and swelling during T helper 1 cell-induced delayed-type hypersensitivity. J. Immunol. 153:3967-3978.

46. Stampfli, M.R., et al. 1999. Interleukin-10 gene transfer to the airway regulates allergic mucosal sensitization in mice. Am. J. Respir. Cell Mol. Biol. 21:586-596.

47. Bellinghausen, I., et al. 2001. Inhibition of human allergic T cell responses by IL-10-treated dendritic cells: differences from hydrocortisone-treated dendritic cells. J. Allergy Clin. Immunol. 108:242-249.

48. Corinti, S., Albanesi, C., la Sala, A., Pastore, S., and Girolomoni, G. 2001 Regulatory activity of autocrine IL-10 on dendritic cell functions. J. Immunol. 166:4312-4318.

49. Huang, L.Y., Reis e Sousa, C., Itoh, Y., Inman, J., and Scott, D.E. 2001. IL-12 induction by a TH1-inducing adjuvant in vivo: dendritic cell subsets and regulation by IL-10. J. Immunol. 167:1423-1430.

50. Liu, L., Rich, B.E., Inobe, J., Chen, W., and Weiner, H.L. 1998. Induction of Th2 cell differentiation in the primary immune response: dendritic cells isolated from adherent cell culture treated with IL-10 prime naive CD4+ T cells to secrete IL-4. Int. Immunol. 10:1017-1026.

51. Umetsu, D.T., McIntire, J.J., Akbari, O., Macaubas, C., and DeKruyff, R.H. 2002. Asthma: an epidemic of dysregulated immunity. Nat. Immunol. 3:715-720.

52. Ohmen, J.D., et al. 1995. Overexpression of IL-10 in atopic dermatitis. Contrasting cytokine patterns with delayed-type hypersensitivity reactions. J. Immunol. 154:1956-1963. 